$>$ Dans leur forme active, liée au GTP, les protéines $G$ de la famille Arf et Sar sont liées aux membranes lipidiques et servent de points d'amarrage aux manteaux protéiques COPI et COPII. Les manteaux protéiques sont des assemblages organisés bidimensionnels qui couvrent les membranes intracellulaires pour les déformer en vésicules de transport et sélectionner les protéines qui doivent être transportées. La forte activité d'hydrolyse du GTP dans les manteaux protéiques pose le problème de la dynamique de ces assemblages et plus précisément du lien entre l'état macroscopique du manteau, assemblé ou désassemblé, et l'état microscopique de la protéine G, liée au GDP ou au GTP. La distribution temporelle et spatiale de la réaction d'hydrolyse du GTP pourrait être déterminante pour le fonctionnement des manteaux. <

\section{Contrôle de l'assemblage des manteaux protéiques COP par les petites protéines G Arf et Sar}

Bruno Antonny



C'est le rôle des manteaux protéiques [1, 2], de larges édifices moléculaires, sortes de polymères de surface, qui s'assemblent sur la face cytosolique des membranes intracellulaires. Ils imposent à la membrane une forte courbure et donnent ainsi naissance à un bourgeon d'un diamètre de moins de $100 \mathrm{~nm}$, précurseur d'une vésicule de transport individualisée ou d'intermédiaires de transport encore mal connus tels que les tubules. II semble que les manteaux concentrent également des protéines destinées à être transportées. Pour des protéines membranaires, cette sélection peut s'effectuer par contact direct avec le manteau. Pour des protéines situées à l'intérieur de la vésicule, la sélection doit faire intervenir des protéines transmembranaires assurant le lien entre le contenant (le manteau) et le contenu.

L'assemblage de la quasi-totalité des manteaux protéiques est contrôlé par un groupe de petites protéines $G$, aux remarquables propriétés d'interaction avec les membranes : les protéines Arf et Sar. Des protéines $G$ classiques, Arf et Sar ont conservé la propriété essentielle de bascule de leur conformation. Toute protéine $G$ adopte deux conformations, l'une liée au GDP et l'autre 
liée au GTP, et seule cette dernière est capable d'interagir avec des protéines cibles [3]. Dans le cas d'Arf et de Sar, la transition GDP/GTP contrôle également l'avidité de la protéine pour les membranes lipidiques. Arf-GDP et Sar-GDP sont essentiellement solubles et sans interaction avec les protéines des manteaux. Arf-GTP et SarGTP sont au contraire fortement attachées aux membranes lipidiques et servent de point d'ancrage aux manteaux $[1,2]$. Le cycle d'assemblage et de désassemblage des manteaux protéiques est donc directement contrôlé par le cycle GDP et GTP des protéines Arf ou Sar. Ce cycle, décrit dans la Figure 1, a été établi au milieu des années 1990 pour deux manteaux : COPI, contrôlé par Arf et assurant le transport rétrograde et antérograde depuis l'appareil de Golgi, et COPII, contrôlé par Sar et assurant le transport depuis le réticulum endoplasmique. Depuis lors, de nombreux progrès ont été accomplis dans notre connaissance des bases structurales de la bascule GDP/GTP d'Arf et de Sar. Moins spectaculaires et parfois plus confus ont été les progrès concernant le lien entre le cycle GDP/GTP d'Arf, l'assemblage des manteaux et la collecte des protéines transportées.



Figure 1. Exemple du contrôle de l'assemblage d'un manteau protéique par une petite protéine $G$ : le manteau COPII. Dans une première étape, la petite protéine G Sar est activée par un facteur d'échange et passe d'une forme GDP soluble à une forme GTP périmembranaire. Sar-GTP sert alors de point d'ancrage aux larges sous-unités du manteau COPII (Sec23/24 [jaune] et Sec13/31 [violet]) qui s'assemblent de manière séquentielle. L'ensemble forme un manteau capable de déformer la membrane en une vésicule transportant des protéines membranaires et des protéines de la lumière. L'hydrolyse du GTP dans Sar est induite par les autres sous-unités du manteau et conduit finalement au désassemblage du manteau.

\section{Bascule GDP/GTP d'Arf et de Sar}

Les protéines $G$ fournissent un bel exemple de variations sur un même thème. Une protéine $G$ est un kit minimal, comprenant 6 brins $\beta$ et 5 hélices $\alpha$ avec, en surface, un site de liaison des nucléotides à guanine composé de quelques acides aminés conservés [3]. Parmi ces résidus, une thréonine et une glycine jouent un rôle particulier. Ces deux résidus forment des liaisons avec le troisième phosphate du GTP, liaisons qui provoquent, lors de l'échange GDP/GTP, un changement de conformation sur des régions plus ou moins étendues appelées switchs. Arf et Sar sont bâties sur ce modèle élémentaire, mais possèdent une région supplémentaire, une hélice amphiphile amino-terminale prolongée, dans le cas d'Arf, par une modification lipidique, un acide gras de 14 carbones. Des études structurales ont montré que, dans la forme GDP, la face hydrophobe de l'hélice est plaquée contre une gorge de la protéine. Sous forme GTP, I'hélice interagit au contraire avec les membranes lipidiques car un changement de conformation spectaculaire du cœur de la protéine $G$ obture la gorge $[4,5]$. La bascule GDP/GTP d'Arf et de Sar est donc une réaction particulièrement complexe, qui couple une substitution de nucléotides à un changement de conformation traversant toute la protéine et libérant un élément essentiellement hydrophobe. Cette réaction spontanément très lente est catalysée par des facteurs d'échange [6]. Indiquons brièvement ici que les facteurs d'échange d'Arf et Sar sont liés aux membranes lipidiques et que cette liaison est essentielle pour leur activité. $\varepsilon$ n effet, même si ces facteurs interagissent non pas avec I'hélice amino-terminale d'Arf ou de Sar mais avec le cœur de la protéine, ils ne peuvent provoquer l'échange GDP/GTP, sans qu'une membrane lipidique n'autorise la libération de cette hélice amino-terminale amphiphile [7]. Dès lors, la formation d'Arf-GTP ou de Sar-GTP sur une membrane est conditionnée par la présence d'un facteur d'échange sur cette même membrane. 


\section{Assemblage d'un manteau et sélection de protéines}

Considérons maintenant une membrane lipidique couverte d'Arf-GTP ou de Sar-GTP. Est-ce une condition suffisante pour provoquer l'assemblage d'un manteau ? La question revient à connaître le degré d'interdépendance entre cet assemblage et la capture de protéines dans les vésicules. Dans une première configuration, le manteau se forme qu'il y ait ou non des protéines à transporter. C'est le modèle de l'autobus qui part à heure fixe avec ou sans passagers. Dans la seconde configuration, l'assemblage du manteau sur une membrane requiert, outre la présence d'Arf-GTP ou de Sar-GTP, des interactions avec l'extrémité cytoplasmique de protéines membranaires. C'est le modèle du taxi, qui ne part qu'avec des clients.

Les travaux du groupe de Randy Schekman ont montré que, sur des liposomes artificiels incubés en présence des petites protéines G Arf ou Sar, d'un analogue non hydrolysable du GTP et des éléments purifiés des manteaux COPI ou COPII, on observe l'apparition d'un manteau dense, visible au microscope électronique, qui couvre les liposomes et provoque le bourgeonnement de petites vésicules $[8,9]$. L'importance de ces résultats réside dans le fait qu'ils suggèrent que l'assemblage des manteaux COP n'est pas strictement dépendant de la présence de protéines membranaires. II est cependant établi que certaines protéines sont concentrées dans les vésicules de transport et qu'il doit exister dans leur domaine cytoplasmique, ou dans celui de leur récepteur, un motif reconnu par le manteau protéique. On sait peu de chose sur cette reconnaissance. De courts motifs tels que la juxtaposition de deux résidus basiques (Lys-Lys en position - 4 et - 3 ) ou la séquence Asp-X-Glu semblent reconnus respectivement par les manteaux COPI et COPII. Le motif Asp-X-Glu est ainsi décisif pour le transport de certains canaux potassiques après leur synthèse dans le réticulum [10].

La membrane de vésicules COP isolées à partir du réticulum endoplasmique ou de l'appareil de Golgi est enrichie de quelques protéines membranaires qui sont sans cesse recyclées entre ces compartiments [11]. Le rôle de ces protéines reste obscur. Dans la levure, leur délétion n'a généralement que des effets mineurs sur le transport global des protéines [12]. L'idée la plus simple est que ces protéines, sans être nécessaires à la formation des vésicules, servent de récepteurs pour enrichir quelques protéines de la lumière dans les vésicules, assurant ainsi un transport effi-
Figure 2. Deux modèles alternatifs mais non exclusifs de l'hydrolyse du GTP au sein d'un manteau COP. Dans le premier modèle (A), l'activité GAP des sous-unités du manteau sur la protéine $G$ est trop importante pour que le manteau atteigne une surface assemblée suffisamment importante. Certaines protéines membranaires exposant un motif d'exportation adéquat auraient un effet inhibiteur sur l'activité GAP, ce qui couplerait stabilisation du manteau et incorporation de protéines. Ici, I'hydrolyse du GTP a un effet négatif à la fois sur la stabilité du manteau et sur la capture de protéines. Le second modèle (B) postule une distribution spatiale de la réaction de telle sorte que subsiste aux marges du manteau - là où les nouvelles sous-unités s'assemblent - la forme GTP d'Arf nécessaire à la stabilité de ces zones. Dans les zones centrales et plus anciennes, l'hydrolyse du GTP a lieu, mais ne provoque pas de désassemblage du fait des multiples contacts latéraux entre unités. C'est une extrapolation du modèle de la tubuline, polymère linéaire, au cas d'un assemblage en deux dimensions. Dans ce modèle, il est possible d'envisager un couplage positif entre hydrolyse du GTP et capture de protéines membranaires si les interactions de ces protéines avec les sous-unités du manteau sont renforcées lors de la bascule GTP/GDP de la protéine $\mathrm{G}$ ou lors de son départ du manteau.

\section{A}

Hydrolyse rapide du GTP : désassemblage prématuré du manteau

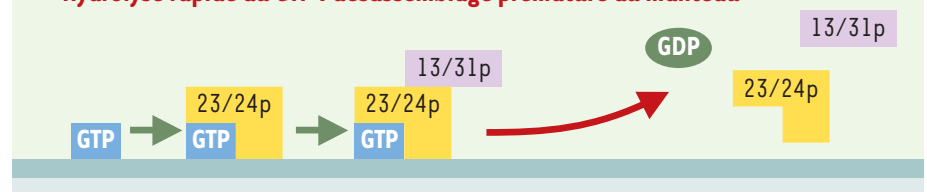

Hydrolyse du GTP ralentie par une protéine membranaire : le manteau s'étend

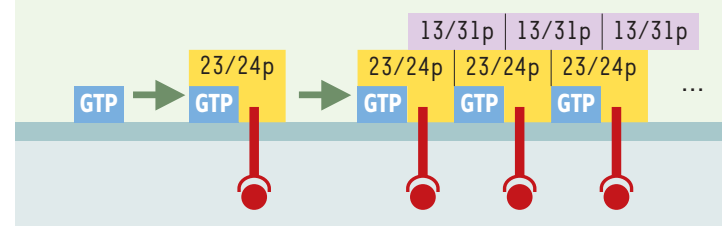

B

Hydrolyse du GTP distribuée dans l'espace : un manteau métastable se forme

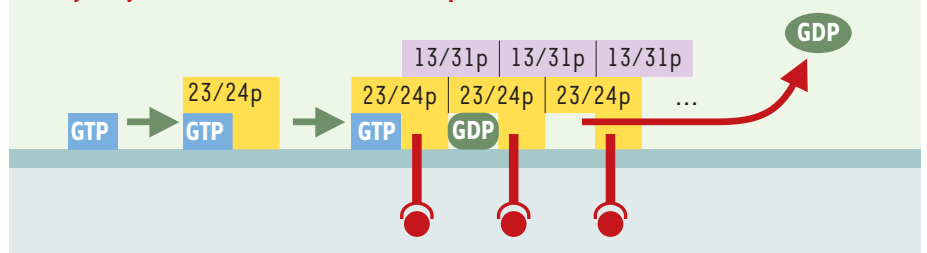


cace. C'est le rôle qui semble par exemple dévolu à la protéine de levure $\varepsilon r v 29 p$. Lorsque cette protéine à 6 segments transmembranaires est supprimée, l'incorporation dans les vésicules COPII d'une phéromone, le facteur $\alpha$, est très fortement réduite, alors que d'autres protéines sont transportées normalement [13].

\section{Le problème de la réaction de GTPase}

Pour le moment, nous n'avons considéré que la réaction a priori la plus noble du cycle d'Arf ou de Sar, celle de l'activation qui aboutit à une forme GTP attachée à la membrane sur laquelle s'assemble un manteau. La réaction d'hydrolyse du GTP semble moins intéressante : revenant à un état GDP, Arf ou Sar se dissocient des membranes, ce qui provoque le désassemblage du manteau. Certes, cette réaction est cruciale parce que la vésicule ne peut fusionner avec un compartiment accepteur tant qu'elle est recouverte d'un manteau $[1,2]$. Mais pour le problème qui nous intéresse, celui de la formation d'une vésicule, les jeux semblent faits dès la formation d'Arf-GTP et de Sar-GTP : le manteau s'assemble, déforme la membrane et concentre des protéines. Pourtant, sans que l'on comprenne pourquoi, la réaction de GTPase pourrait avoir un rôle déterminant dès ces premières étapes.

La réaction d'hydrolyse du GTP dans un manteau est problématique pour deux raisons : le caractère polymérique du manteau et le fait que la réaction de GTPase dans Arf ou Sar est fortement stimulée par le manteau. Ainsi, si la protéine GAP qui stimule l'hydrolyse du GTP couplé à Arf n'est pas un élément du manteau COPI, son activité est fortement augmentée par le coatomer, le large complexe protéique qui forme ce manteau [14]. Dans le cas du manteau COPII, la protéine GAP de Sar est l'une des sous-unités du manteau ( $\operatorname{Sec} 23 p$ ) et son activité est décuplée lorsque les autres éléments de ce manteau s'assemblent [15]. Bref, le ver est dans le fruit et l'on peut se demander comment un manteau d'une taille suffisante peut se former si, pendant que des unités élémentaires s'assemblent, d'autres plus anciennes se dissocient en raison de leur activité GAP.

Faut-il alors freiner cette réaction de GTPase ? L'idée est séduisante : une protéine transportée, ou son récepteur, réduirait l'effet du manteau sur la réaction de GTPase, ce qui, en retour, stabiliserait le manteau et augmenterait la probabilité de capture de la protéine dans une vésicule (Figure 2A). Reste qu'il s'agit d'une hypothèse qui n'a été testée que dans un système purement soluble ne mimant pas la situation d'un manteau s'assemblant sur une membrane [16].

À l'opposé de ce modèle, certaines expériences suggèrent que la réaction d'hydrolyse du GTP a un rôle positif pour la capture d'une protéine dans une vésicule [17, 18]. Les vésicules COPI produites dans des conditions où la GTPase est bloquée semblent particulièrement pauvres en contenu, c'est-à-dire en protéines transportées, par rapport à des vésicules où la réaction de GTPase est permise. On se retrouve donc devant le dilemme suivant : le modèle classique prédit que le ralentissement voire le blocage de la GTPase, avantage l'incorporation de protéines cargos, alors que des expériences suggèrent que la GTPase est nécessaire à cette incorporation.

La clé du problème pourrait résider dans la distribution spatiale et temporelle de la réaction de GTPase (Figure 2B). Les manteaux protéiques ne sont pas les seuls polymères fonctionnant à partir d'unités élémentaires oscillant entre deux conformations GTP et GDP. L'exemple le mieux connu est la tubuline qui se polymérise en microtubule. Le paradoxe apparent de ce polymère est que, si seule la forme GTP de la tubuline se polymérise, un microtubule est en fait essentiellement composé de tubuline sous forme GDP [19]. Chaque sous-unité de tubuline GTP qui s'assemble à l'extrémité d'un microtubule agit en effet comme GAP sur la sous-unité précédente. Grâce à ce mécanisme où la réaction de GTPase est distribuée dans l'espace, on obtient un polymère métastable : le dernier anneau de tubuline GTP contraint les autres sous-unités sous forme GDP à rester dans le microtubule. Cet exemple suggère que, dans un manteau, I'hydrolyse du GTP n'est pas nécessairement synonyme de désassemblage immédiat (Figure 2B). II resterait ensuite à montrer pourquoi, dans un manteau où coexisteraient les formes GTP et GDP d'Arf ou de Sar, la sélection de protéines membranaires serait plus efficace que dans un manteau comprenant uniquement la forme GTP. La découverte toute récente d'une interaction préférentielle entre une protéine membranaire, p23, caractéristique des vésicules COP et la forme GDP d'Arf est, à cet égard, particulièrement troublante [20]. $\diamond$

\section{SUMMARY}

\section{COP coats and their control}

by the small G proteins Arf and Sar

In the GTP-bound state, the small G proteins Arf and Sar act as membrane anchors for the assembly of protein coats such as COPI and COPII. Protein coats are regular 2D assembly, which cover intracellular membranes, select cargoes, and drive the formation of transport vesicles. Very fast GTP hydrolysis occurs in Arf and Sar upon COP coat assembly. This raises the problem of the dynamics of COP coats, that is the intimate link between the stability of the polymer and the nucleotide state of Arf or Sar. The temporal and spatial distribution of the GTP hydrolysis reaction is probably a key determinant for the correct assembly and functioning of COP coats. $\diamond$ 


\section{RÉFÉRENCES}

1. Schekman R, Orci L. Coat proteins and vesicle budding. Science 1996 ; 271 : 1526-33.

2. Rothman JE, Wieland FT. Protein sorting by transport vesicles. Science 1996 ; 272 : 227-34

3. Vetter IR, Wittinghofer $A$. The guanine nucleotide-binding switch in three dimensions. Science 2001 ; 294 : 1299-304.

4. Antonny B, Beraud-Dufour $S$, Chardin P, Chabre M. Nterminal hydrophobic residues of the $\mathrm{G}$-protein ADP-ribosylation factor-1 insert into membrane phospholipids upon GDP to GTP exchange. Biochemistry 1997 ; 36 : 4675-84.

5. Goldberg J. Structural basis for activation of ARF GTPase: mechanisms of guanine nucleotide exchange and GTP-myristoyl switching. Cell 1998 ; 95 : 237-48.

6. Chardin P, Antonny B, Cherfils J. Activation des petites protéines $G$ par leur facteur d'échange. Med Sci

$2000 ; 16: 228-34$.

7. Béraud-Dufour $S$, Paris $S$, Chabre M, Antonny B. Dual interaction of ADP ribosylation factor 1 with $\mathrm{Sec} 7$ domain and with lipid membranes during catalysis of guanine nucleotide exchange. J Biol Chem 1999 ; $274: 37629-36$.

8. Matsuoka K, Orci L, Amherdt $M$, et al. COPII-coated vesicle formation reconstituted with purified coat proteins and chemically defined liposomes. Cell 1998 ; 93 : 263-75.

9. Spang A, Matsuoka K, Hamamoto S, Schekman R, Orci L. Coatomer, Arflp, and nucleotide are required to bud coat protein complex Icoated vesicles from large synthetic liposomes. Proc Natl Acad Sci USA 1998 ; 95 : 11199-204.

10. Ma D, Zerangue N, Lin YF, et al. Role of $\varepsilon R$ export signals in controlling surface potassium channel numbers. Science 2001 ; 291 : 316-9.

11. Otte S, Belden WJ, Heidtman
M, Liu J, Jensen ON, Barlowe C. Erv4lp and Erv46p: new components of COPII vesicles involved in transport between the ER and Golgi complex. J Cell Biol 2001; 152 : 503-18.

12. Springer $S$, Chen $\varepsilon$, Duden $R$, et al. The p24 proteins are not essential for vesicular transport in Saccharomyces cerevisiae. Proc Natl Acad Sci USA 2000 ; 97 : 4034-9.

13. Belden WJ, Barlowe C. Role of Erv29p in collecting soluble secretory proteins into $\varepsilon R$ derived transport vesicles. Science 2001 ; 294 :

1528-31.

14. Goldberg J. Structural and functional analysis of the ARFl-ARFGAP complex reveals a role for coatomer in GTP hydrolysis. Cell 1999 ; 96: 893-902.

15. Antonny B, Madden D, Hamamoto S, Orci L, Schekman R. Dynamics of the COPII coat with GTP and stable analogues. Nat Cell Biol 2001 ; 3 : 531-7.

16. Goldberg J. Decoding of sorting signals by coatomer through a GTPase switch in the COPI coat complex. Cell $2000 ; 100: 671-9$.

17. Lanoix J, Ouwendijk J, Stark $A$, et al. Sorting of Golgi resident proteins into different subpopulations of COPI vesicles: a role for ArfGAPl. J Cell Biol 2001 ; 155 : 1199-212.

18. Malsam J, Gommel D, Wieland FT, Nickel W. A role for ADP ribosylation factor in the control of cargo uptake during COPI-coated vesicle biogenesis. FEBS Lett 1999 ; 462 : 267-72.

19. Nogales $\varepsilon$. Structural insight into microtubule function. Annu Rev Biophys Biomol Struct 2001 ; 30 : 397-420.

20. Gommel DU, Memon AR, Heiss $A$, et al. Recruitment to Golgi membranes of ADPribosylation factor $l$ is mediated by the cytoplasmic domain of p23. EMBO J $2001 ; 20: 6751-60$

TIRÉS À PART

B. Antonny 